Article

\title{
Hankel and Toeplitz Determinants for a Subclass of q-Starlike Functions Associated with a General Conic Domain
}

\author{
Hari M. Srivastava ${ }^{1,2, *(0)}$, Qazi Zahoor Ahmad ${ }^{3}$, Nasir Khan ${ }^{4}$, Nazar Khan ${ }^{3}$ and Bilal Khan ${ }^{3}(\mathbb{0}$ \\ 1 Department of Mathematics and Statistics, University of Victoria, Victoria, BC V8W 3R4, Canada \\ 2 Department of Medical Research, China Medical University Hospital, China Medical University, \\ Taichung 40402, Taiwan \\ 3 Department of Mathematics, Abbottabad University of Science and Technology, Abbottabad 22010, Pakistan; \\ zahoorqazi5@gmail.com (Q.Z.A.); nazarmaths@gmail.com (N.K.); bilalmaths789@gmail.com (B.K.) \\ 4 Department of Mathematics, FATA University, Akhorwal (Darra Adam Khel), FR Kohat 26000, Pakistan; \\ dr.nasirkhan@fu.edu.pk \\ * Correspondence: harimsri@math.uvic.ca
}

Received: 29 January 2019; Accepted: 12 February 2019; Published: 15 February 2019

\begin{abstract}
By using a certain general conic domain as well as the quantum (or $q$-) calculus, here we define and investigate a new subclass of normalized analytic and starlike functions in the open unit disk $\mathbb{U}$. In particular, we find the Hankel determinant and the Toeplitz matrices for this newly-defined class of analytic $q$-starlike functions. We also highlight some known consequences of our main results.

Keywords: analytic functions; starlike and $q$-starlike functions; $q$-derivative operator; $q$-hypergeometric functions; conic and generalized conic domains; Hankel determinant; Toeplitz matrices
\end{abstract}

MSC: Primary 05A30, 30C45; Secondary 11B65, 47B38

\section{Introduction and Definitions}

Let the class of functions, which are analytic in the open unit disk

$$
\mathbb{U}=\{z: z \in \mathbb{C} \text { and }|z|<1\},
$$

be denoted by $\mathcal{L}(\mathbb{U})$. Also let $\mathcal{A}$ denote the class of all functions $f$, which are analytic in the open unit disk $\mathbb{U}$ and normalized by

$$
f(0)=0 \text { and } f^{\prime}(0)=1 .
$$

Then, clearly, each $f \in \mathcal{A}$ has a Taylor-Maclaurin series representation as follows:

$$
f(z)=z+\sum_{n=2}^{\infty} a_{n} z^{n} \quad(z \in \mathbb{U}) .
$$

Suppose that $\mathcal{S}$ is the subclass of the analytic function class $\mathcal{A}$, which consists of all functions which are also univalent in $\mathbb{U}$.

A function $f \in \mathcal{A}$ is said to be starlike in $\mathbb{U}$ if it satisfies the following inequality:

$$
\Re\left(\frac{z f^{\prime}(z)}{f(z)}\right)>0 \quad(z \in \mathbb{U}) .
$$


We denote by $\mathcal{S}^{*}$ the class of all such starlike functions in $\mathbb{U}$.

For two functions $f$ and $g$, analytic in $\mathbb{U}$, we say that the function $f$ is subordinate to the function $g$ and write this subordination as follows:

$$
f \prec g \quad \text { or } \quad f(z) \prec g(z) \text {, }
$$

if there exists a Schwarz function $w$ which is analytic in $\mathbb{U}$, with

$$
w(0)=0 \quad \text { and } \quad|w(z)|<1,
$$

such that

$$
f(z)=g(w(z)) .
$$

In the case when the function $g$ is univalent in $\mathbb{U}$, then we have the following equivalence (see, for example, [1]; see also [2]):

$$
f(z) \prec g(z) \quad(z \in \mathbb{U}) \Longleftrightarrow f(0)=g(0) \text { and } f(\mathbb{U}) \subset g(\mathbb{U}) .
$$

Next, for a function $f \in \mathcal{A}$ given by (1) and another function $g \in \mathcal{A}$ given by

$$
g(z)=z+\sum_{n=2}^{\infty} b_{n} z^{n} \quad(z \in \mathbb{U})
$$

the convolution (or the Hadamard product) of $f$ and $g$ is defined here by

$$
(f * g)(z):=z+\sum_{n=2}^{\infty} a_{n} b_{n} z^{n}=:(g * f)(z) .
$$

Let $\mathcal{P}$ denote the well-known Carathéodory class of functions $p$, analytic in the open unit disk $\mathbb{U}$, which are normalized by

$$
p(z)=1+\sum_{n=1}^{\infty} c_{n} z^{n}
$$

such that

$$
\Re(p(z))>0 \quad(z \in \mathbb{U}) .
$$

Following the works of Kanas et al. (see [3,4]; see also [5]), we introduce the conic domain $\Omega_{k}$ $(k \geqq 0)$ as follows:

$$
\Omega_{k}=\left\{u+i v: u>k \sqrt{(u-1)^{2}+v^{2}}\right\} .
$$

In fact, subjected to the conic domain $\Omega_{k}(k \geqq 0)$, Kanas and Wiśniowska (see [3,4]; see also [6]) studied the corresponding class $k-\mathcal{S} \mathcal{T}$ of $k$-starlike functions in $\mathbb{U}$ (see Definition 1 below). For fixed $k, \Omega_{k}$ represents the conic region bounded successively by the imaginary axis $(k=0)$, by a parabola $(k=1)$, by the right branch of a hyperbola $(0<k<1)$, and by an ellipse $(k>1)$.

For these conic regions, the following functions play the role of extremal functions. 


$$
p_{k}(z)= \begin{cases}\frac{1+z}{1-z}=1+2 z+2 z^{2}+\cdots & (k=0) \\ 1+\frac{2}{\pi^{2}}\left[\log \left(\frac{1+\sqrt{z}}{1-\sqrt{z}}\right)\right]^{2} & (k=1) \\ 1+\frac{2}{1-k^{2}} \sinh ^{2}\left[\left(\frac{2}{\pi} \arccos k\right) \arctan (h \sqrt{z})\right] & (0 \leqq k<1) \\ 1+\frac{1}{k^{2}-1}\left[1+\sin \left(\frac{\pi}{2 K(\kappa)} \int_{0}^{\frac{u(z)}{\sqrt{k}}} \frac{d t}{\left.\left.\sqrt{\left(1-t^{2}\right)\left(1-\kappa^{2} t^{2}\right)}\right)\right]}\right.\right. & (k>1),\end{cases}
$$

where

$$
u(z)=\frac{z-\sqrt{\kappa}}{1-\sqrt{\kappa} z} \quad(z \in \mathbb{U})
$$

and $\kappa \in(0,1)$ is so chosen that

$$
k=\cosh \left(\frac{\pi K^{\prime}(\kappa)}{4 K(\kappa)}\right) .
$$

Here $K(\kappa)$ is Legendre's complete elliptic integral of first kind and

$$
K^{\prime}(\kappa)=K\left(\sqrt{1-\kappa^{2}}\right)
$$

that is, $K^{\prime}(\kappa)$ is the complementary integral of $K(\kappa)$ (see, for example, ([7], p. 326, Equation $\left.9.4(209)\right)$ ). Indeed, from (5), we have

$$
p_{k}(z)=1+p_{1} z+p_{2} z^{2}+p_{3} z^{3}+\cdots .
$$

The class $k-\mathcal{S} \mathcal{T}$ is defined as follows.

Definition 1. A function $f \in \mathcal{A}$ is said to be in the class $k-\mathcal{S} \mathcal{T}$ if and only if

$$
\frac{z f^{\prime}(z)}{f(z)} \prec p_{k}(z) \quad(\forall z \in \mathbb{U} ; k \geqq 0) .
$$

We now recall some basic definitions and concept details of the $q$-calculus which will be used in this paper (see, for example, ([7], p. 346 et seq.)). Throughout the paper, unless otherwise mentioned, we suppose that $0<q<1$ and

$$
\mathbb{N}=\{1,2,3 \cdots\}=\mathbb{N}_{0} \backslash\{0\} \quad\left(\mathbb{N}_{0}:=\{0,1,2, \cdots\}\right) .
$$

Definition 2. Let $q \in(0,1)$ and define the $q$-number $[\lambda]_{q}$ by

$$
[\lambda]_{q}= \begin{cases}\frac{1-q^{\lambda}}{1-q} & (\lambda \in \mathbb{C}) \\ \sum_{k=0}^{n-1} q^{k}=1+q+q^{2}+\cdots+q^{n-1} & (\lambda=n \in \mathbb{N}) .\end{cases}
$$

Definition 3. Let $q \in(0,1)$ and define the $q$-factorial $[n]_{q} !$ by

$$
[n]_{q} != \begin{cases}1 & (n=0) \\ \prod_{k=1}^{n}[k]_{q} & (n \in \mathbb{N}) .\end{cases}
$$


Definition 4 (see $[8,9])$. The $q$-derivative (or $q$-difference) operator $D_{q}$ of a function $f$ defined, in a given subset of $\mathbb{C}$, by

$$
\left(D_{q} f\right)(z)= \begin{cases}\frac{f(z)-f(q z)}{(1-q) z} & (z \neq 0) \\ f^{\prime}(0) & (z=0),\end{cases}
$$

provided that $f^{\prime}(0)$ exists.

From Definition 4, we can observe that

$$
\lim _{q \rightarrow 1-}\left(D_{q} f\right)(z)=\lim _{q \rightarrow 1-} \frac{f(z)-f(q z)}{(1-q) z}=f^{\prime}(z)
$$

for a differentiable function $f$ in a given subset of $\mathbb{C}$. It is also known from (1) and (7) that

$$
\left(D_{q} f\right)(z)=1+\sum_{n=2}^{\infty}[n]_{q} a_{n} z^{n-1} .
$$

Definition 5. The q-Pochhammer symbol $[\xi]_{n, q}\left(\xi \in \mathbb{C} ; n \in \mathbb{N}_{0}\right)$ is defined as follows:

$$
[\xi]_{n, q}=\frac{\left(q^{\xi} ; q\right)_{n}}{(1-q)^{n}}= \begin{cases}1 & (n=0) \\ {[\xi]_{q}[\xi+1]_{q}[\xi+2]_{q} \cdots[\xi+n-1]_{q}} & (n \in \mathbb{N}) .\end{cases}
$$

Moreover, the q-gamma function is defined by the following recurrence relation:

$$
\Gamma_{q}(z+1)=[z]_{q} \Gamma_{q}(z) \quad \text { and } \quad \Gamma_{q}(1)=1 .
$$

Definition 6 (see [10]). For $f \in \mathcal{A}$, let the $q$-Ruscheweyh derivative operator $\mathcal{R}_{q}^{\lambda}$ be defined, in terms of the Hadamard product (or convolution) given by (2), as follows:

$$
\mathcal{R}_{q}^{\lambda} f(z)=f(z) * \mathcal{F}_{q, \lambda+1}(z) \quad(z \in \mathbb{U} ; \lambda>-1),
$$

where

$$
\mathcal{F}_{q, \lambda+1}(z)=z+\sum_{n=2}^{\infty} \frac{\Gamma_{q}(\lambda+n)}{[n-1]_{q} ! \Gamma_{q}(\lambda+1)} z^{n}=z+\sum_{n=2}^{\infty} \frac{[\lambda+1]_{q, n-1}}{[n-1]_{q} !} z^{n}
$$

We next define a certain $q$-integral operator by using the same technique as that used by Noor [11].

Definition 7. For $f \in \mathcal{A}$, let the q-integral operator $\mathcal{F}_{q, \lambda}$ be defined by

$$
\mathcal{F}_{q, \lambda+1}^{-1}(z) * \mathcal{F}_{q, \lambda+1}(z)=z\left(D_{q} f\right)(z)
$$

Then

$$
\begin{aligned}
\mathcal{I}_{q}^{\lambda} f(z) & =f(z) * \mathcal{F}_{q, \lambda+1}^{-1}(z) \\
& =z+\sum_{n=2}^{\infty} \psi_{n-1} a_{n} z^{n} \quad(z \in \mathbb{U} ; \lambda>-1),
\end{aligned}
$$

where

$$
\mathcal{F}_{q, \lambda+1}^{-1}(z)=z+\sum_{n=2}^{\infty} \psi_{n-1} z^{n}
$$


and

$$
\psi_{n-1}=\frac{[n]_{q} ! \Gamma_{q}(\lambda+1)}{\Gamma_{q}(\lambda+n)}=\frac{[n]_{q} !}{[\lambda+1]_{q, n-1}}
$$

Clearly, we have

$$
\mathcal{I}_{q}^{0} f(z)=z\left(D_{q} f\right)(z) \quad \text { and } \quad \mathcal{I}_{q}^{1} f(z)=f(z)
$$

We note also that, in the limit case when $q \rightarrow 1-$, the $q$-integral operator $\mathcal{F}_{q, \lambda}$ given by Definition 7 would reduce to the integral operator which was studied by Noor [11].

The following identity can be easily verified:

$$
z D_{q}\left(\mathcal{I}_{q}^{\lambda+1} f(z)\right)=\left(1+\frac{[\lambda]_{q}}{q^{\lambda}}\right) \mathcal{I}_{q}^{\lambda} f(z)-\frac{[\lambda]_{q}}{q^{\lambda}} \mathcal{I}_{q}^{\lambda+1} f(z)
$$

When $q \rightarrow 1-$, this last identity in (10) implies that

$$
z\left(\mathcal{I}^{\lambda+1} f(z)\right)^{\prime}=(1+\lambda) \mathcal{I}^{\lambda} f(z)-\lambda \mathcal{I}^{\lambda+1} f(z)
$$

which is the well-known recurrence relation for the above-mentioned integral operator which was studied by Noor [11].

In geometric function theory, several subclasses belonging to the class of normalized analytic functions class $\mathcal{A}$ have already been investigated in different aspects. The above-defined $q$-calculus gives valuable tools that have been extensively used in order to investigate several subclasses of $\mathcal{A}$. Ismail et al. [12] were the first who used the $q$-derivative operator $D_{q}$ to study the $q$-calculus analogous of the class $\mathcal{S}^{*}$ of starlike functions in $\mathbb{U}$ (see Definition 8 below). However, a firm footing of the $q$-calculus in the context of geometric function theory was presented mainly and basic (or $q$-) hypergeometric functions were first used in geometric function theory in a book chapter by Srivastava (see, for details, ([13], p. 347 et seq.); see also [14]).

Definition 8 (see [12]). A function $f \in \mathcal{A}$ is said to belong to the class $\mathcal{S}_{q}^{*}$ if

$$
f(0)=f^{\prime}(0)-1=0
$$

and

$$
\left|\frac{z}{f(z)}\left(D_{q} f\right) z-\frac{1}{1-q}\right| \leqq \frac{1}{1-q}
$$

It is readily observed that, as $q \rightarrow 1-$, the closed disk:

$$
\left|w-\frac{1}{1-q}\right| \leqq \frac{1}{1-q}
$$

becomes the right-half plane and the class $\mathcal{S}_{q}^{*}$ of $q$-starlike functions reduces to the familiar class $\mathcal{S}^{*}$ of normalized starlike functions in $\mathbb{U}$ with respect to the origin $(z=0)$. Equivalently, by using the principle of subordination between analytic functions, we can rewrite the conditions in (11) and (12) as follows (see [15]):

$$
\frac{z}{f(z)}\left(D_{q} f\right)(z) \prec \widehat{p}(z) \quad\left(\widehat{p}(z)=\frac{1+z}{1-q z}\right) .
$$

The notation $\mathcal{S}_{q}^{*}$ was used by Sahoo and Sharma [16].

Now, making use of the principle of subordination between analytic functions and the above-mentioned $q$-calculus, we present the following definition. 
Definition 9. A function $p$ is said to be in the class $k-\mathcal{P}_{q}$ if and only if

$$
p(z) \prec \frac{2 p_{k}(z)}{(1+q)+(1-q) p_{k}(z)}
$$

where $p_{k}(z)$ is defined by (5).

Geometrically, the function $p(z) \in k-\mathcal{P}_{q}$ takes on all values from the domain $\Omega_{k, q}(k \geqq 0)$ which is defined as follows:

$$
\Omega_{k, q}=\left\{w: \Re\left(\frac{(1+q) w}{(q-1) w+2}\right)>k\left|\frac{(1+q) w}{(q-1) w+2}-1\right|\right\} .
$$

The domain $\Omega_{k, q}$ represents a generalized conic region.

It can be seen that

$$
\lim _{q \rightarrow 1-} \Omega_{k, q}=\Omega_{k}
$$

where $\Omega_{k}$ is the conic domain considered by Kanas and Wiśniowska [3]. Below, we give some basic facts about the class $k-\mathcal{P}_{q}$.

Remark 1. First of all, we see that

$$
k-\mathcal{P}_{q} \subseteq \mathcal{P}\left[\frac{2 k}{2 k+1+q}\right],
$$

where $\mathcal{P}\left[\frac{2 k}{2 k+1+q}\right]$ is the well-known class of functions with real part greater than $\frac{2 k}{2 k+1+q}$. Secondly, we have

$$
\lim _{q \rightarrow 1-} k-\mathcal{P}_{q}=\mathcal{P}\left(p_{k}\right),
$$

where $\mathcal{P}\left(p_{k}\right)$ is the well-known function class introduced by Kanas and Wiśniowska [3]. Thirdly, we have

$$
\lim _{q \rightarrow 1-} 0-\mathcal{P}_{q}=\mathcal{P},
$$

where $\mathcal{P}$ is the well-known class of analytic functions with positive real part.

Definition 10. A function $f$ is said to be in the class $\mathcal{S T}(k, \lambda, q)$ if and only if

$$
\frac{z\left(D_{q} \mathcal{I}_{q}^{\lambda} f\right)(z)}{f(z)} \in k-\mathcal{P}_{q} \quad(k \geqq 0 ; \lambda \geqq 0),
$$

or, equivalently,

$$
\Re\left(\frac{(1+q) \frac{z\left(D_{q} \mathcal{I}_{q}^{\lambda} f\right)(z)}{f(z)}}{(q-1) \frac{z\left(D_{q} \mathcal{I}_{q}^{\lambda} f\right)(z)}{f(z)}+2}\right)>k\left|\frac{(1+q) \frac{z\left(D_{q} \mathcal{I}_{q}^{\lambda} f\right)(z)}{f(z)}}{(q-1) \frac{z\left(D_{q} \mathcal{I}_{q}^{\lambda} f\right)(z)}{f(z)}+2}-1\right| .
$$

Remark 2. First of all, it is easily seen that

$$
\mathcal{S T}(0,1, q)=\mathcal{S}_{q}^{*},
$$

where $\mathcal{S}_{q}^{*}$ is the function class introduced and studied by Ismail et al. [12]. Secondly, we have

$$
\lim _{q \rightarrow 1-} \mathcal{S T}(k, 1, q)=k-\mathcal{S} \mathcal{T}
$$


where $k-\mathcal{S} \mathcal{T}$ is a function class introduced and studied by Kanas and Wiśniowska [4]. Finally, we have

$$
\lim _{q \rightarrow 1-} \mathcal{S} \mathcal{T}(0,1, q)=\mathcal{S}^{*}
$$

where $\mathcal{S}^{*}$ is the well-known class of starlike functions in $\mathbb{U}$ with respect to the origin $(z=0)$.

Remark 3. Further studies of the new $q$-starlike function class $\mathcal{S T}(k, \lambda, q)$, as well as of its more consequences, can next be determined and investigated in future papers.

Let $n \in \mathbb{N}_{0}$ and $j \in \mathbb{N}$. The following $j$ th Hankel determinant was considered by Noonan and Thomas [17]:

$$
\mathcal{H}_{j}(n)=\left|\begin{array}{lllll}
a_{n} & a_{n+1} & \cdot & \cdot & a_{n+j-1} \\
a_{n+1} & \cdot & & \cdot \\
\cdot & \cdot & & \cdot \\
\cdot & \cdot & & \cdot \\
\cdot & \cdot & & \cdot \\
a_{n+j-1} & \cdot & \cdot & \cdot & a_{n+2(j-1)}
\end{array}\right|,
$$

where $a_{1}=1$. In fact, this determinant has been studied by several authors, and sharp upper bounds on $\mathcal{H}_{2}$ (2) were obtained by several authors (see [18-20]) for various classes of functions. It is well-known that the Fekete-Szegö functional $\left|a_{3}-a_{2}^{2}\right|$ can be represented in terms of the Hankel determinant as $\mathcal{H}_{2}(1)$. This functional has been further generalized as $\left|a_{3}-\mu a_{2}^{2}\right|$ for some real or complex $\mu$. Fekete and Szegö gave sharp estimates of $\left|a_{3}-\mu a_{2}^{2}\right|$ for $\mu$ real and $f \in \mathcal{S}$, the class of normalized univalent functions in $\mathbb{U}$. It is also known that the functional $\left|a_{2} a_{4}-a_{3}^{2}\right|$ is equivalent to $\mathcal{H}_{2}(2)$ (see [18]). Babalola [21] studied the Hankel determinant $\mathcal{H}_{3}(1)$ for some subclasses of normalized analytic functions in $\mathbb{U}$. The symmetric Toeplitz determinant $\mathcal{T}_{j}(n)$ is defined by

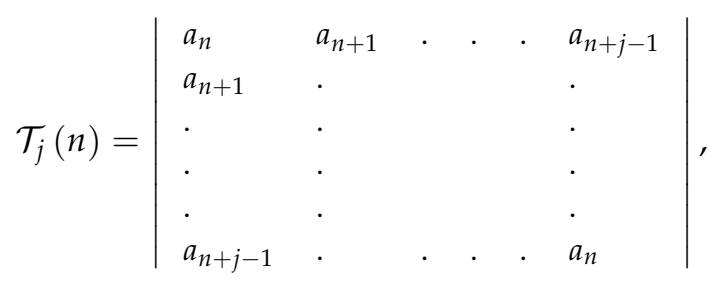

so that

$$
\mathcal{T}_{2}(2)=\left|\begin{array}{ll}
a_{2} & a_{3} \\
a_{3} & a_{2}
\end{array}\right|, \quad \mathcal{T}_{2}(3)=\left|\begin{array}{ll}
a_{3} & a_{4} \\
a_{4} & a_{3}
\end{array}\right|, \quad \mathcal{T}_{3}(2)=\left|\begin{array}{lll}
a_{2} & a_{3} & a_{4} \\
a_{3} & a_{2} & a_{3} \\
a_{4} & a_{3} & a_{2}
\end{array}\right|,
$$

and so on.

For $f \in \mathcal{S}$, the problem of finding the best possible bounds for ||$a_{n+1}|-| a_{n}||$ has a long history (see, for details, [22]). It is a known fact from [22] that

$$
|| a_{n+1}|-| a_{n}||<c
$$

for a constant $c$. However, the problem of finding exact values of the constant $c$ for $\mathcal{S}$ and its various subclasses has proved to be difficult. In a very recent investigation, Thomas and Abdul-Halim [23] succeeded in obtaining some sharp estimates for $\mathcal{T}_{j}(n)$ for the first few values of $n$ and $j$ involving symmetric Toeplitz determinants whose entries are the coefficients $a_{n}$ of starlike and close-toconvex functions. 
In the present investigation, our focus is on the Hankel determinant and the Toeplitz matrices for the function class $\mathcal{S} \mathcal{T}(k, \lambda, q)$ given by Definition 10.

\section{A Set of Lemmas}

In order to prove our main results in this paper, we need each of the following lemmas.

Lemma 1 (see [20]). If the function $p(z)$ given by (3) is in the Carathéodory class $\mathcal{P}$ of analytic functions with positive real part in $\mathbb{U}$, then

$$
2 c_{2}=c_{1}^{2}+x\left(4-c_{1}^{2}\right)
$$

and

$$
4 c_{3}=c_{1}^{3}+2\left(4-c_{1}^{2}\right) c_{1} x-c_{1}\left(4-c_{1}^{2}\right) x^{2}+2\left(4-c_{1}^{2}\right)\left(1-\left|x^{2}\right|\right) z
$$

for some $x, z \in \mathbb{C}$ with $|x| \leqq 1$ and $|z| \leqq 1$.

Lemma 2 (see [24]). Let the function $p(z)$ given by (3) be in the Carathéodory class $\mathcal{P}$ of analytic functions with positive real part in $\mathbb{U}$. Also let $\mu \in \mathbb{C}$. Then

$$
\left|c_{n}-\mu c_{k} c_{n-k}\right| \leqq 2 \max (1,|2 \mu-1|) \quad(1 \leqq k \leqq n-1) .
$$

Lemma 3 (see [22]). Let the function $p(z)$ given by (3) be in the Carathéodory class $\mathcal{P}$ of analytic functions with positive real part in $\mathbb{U}$. Then

$$
\left|c_{n}\right| \leqq 2 \quad(n \in \mathbb{N}) .
$$

This last inequality is sharp.

\section{Main Results}

Throughout this section, unless otherwise mentioned, we suppose that

$$
q \in(0,1), \lambda>-1 \text { and } k \in[0,1] \text {. }
$$

Theorem 1. If the function $f(z)$ given by (1) belongs to the class $\mathcal{S T}(k, \lambda, q)$, where $k \in[0,1]$, then

$$
\begin{gathered}
\left|a_{2}\right| \leqq \frac{(1+q) p_{1}}{2 q \psi_{1}} \\
a_{3} \leqq \frac{1}{2 q \psi_{2}}\left(p_{1}+\left|p_{2}-p_{1}+\frac{\left(q^{2}+1\right) p_{1}^{2}}{2 q}\right|\right)
\end{gathered}
$$

and

$$
\begin{aligned}
a_{4} \leqq & \frac{(1+q)}{4\left(q+q^{2}+q^{3}\right) \psi_{3}}\left(2 p_{1}+4\left|p_{2}-p_{1}+\frac{\left(2+q^{2}\right) p_{1}^{2}}{4 q}\right|\right. \\
& +\mid 2 p_{3}+2 p_{1}-4 p_{2}-\frac{\left(2\left(1+q^{2}\right)-q\right) p_{1}^{2}}{q}+\frac{\left(4 q^{2}-3 q+2\right)}{q} p_{1} p_{2} \\
& \left.+\frac{\left(q^{2}+2 q-1\right)}{2 q^{2}} p_{1}^{3} \mid\right)
\end{aligned}
$$

where $p_{j}(j=1,2,3)$ are positive and are the coefficients of the functions $p_{k}(z)$ defined by (6). Each of the above results is sharp for the function $g(z)$ given by

$$
g(z)=\frac{2 p_{k}(z)}{(1+q)+(1-q) p_{k}(z)} .
$$


Proof. Let $f(z) \in \mathcal{S} \mathcal{T}(k, \lambda, q)$. Then, we have

$$
\frac{z\left(D_{q} f\right)(z)}{f(z)}=\mathfrak{q}(z) \prec S_{k}(z),
$$

where

$$
S_{k}(z)=\frac{2 p_{k}(z)}{(1+q)+(1-q) p_{k}(z)},
$$

and the functions $p_{k}(z)$ are defined by (6).

We now define the function $p(z)$ with $p(0)=1$ and with a positive real part in $\mathbb{U}$ as follows:

$$
p(z)=\frac{1+S_{k}^{-1}(\mathfrak{q}(z))}{1-S_{k}^{-1}(\mathfrak{q}(z))}=1+c_{1} z+c_{2} z^{2}+\cdots
$$

After some simple computation involving (16), we get

$$
\mathfrak{q}(z)=S_{k}\left(\frac{p(z)+1}{p(z)-1}\right) .
$$

We thus find that

$$
\begin{aligned}
S_{k}\left(\frac{p(z)+1}{p(z)-1}\right) & \\
=1 & +\left(\frac{q+1}{2}\right)\left[\frac{p_{1} c_{1}}{2} z+\left\{\frac{p_{1} c_{2}}{2}+\left(\frac{p_{2}}{4}-\frac{p_{1}}{4}+\left(\frac{(q-1) p_{1}^{2}}{8}\right)\right) c_{1}^{2}\right\} z^{2}\right. \\
& +\left\{\frac{p_{1} c_{3}}{2}+\left(\frac{p_{2}}{2}-\frac{p_{1}}{2}+\left(\frac{(q-1) p_{1}^{2}}{4}\right)\right) c_{1} c_{2}\right. \\
& \left.\left.+\left(\frac{p_{1}}{8}-\frac{p_{2}}{4}-\frac{(q-1) p_{1}^{2}}{8}+\frac{p_{3}}{8}-\frac{(q-1) p_{1} p_{2}}{8}+\frac{(q-1)^{2} p_{1}^{3}}{32}\right) c_{1}^{3}\right\} z^{3}\right]+\cdots .
\end{aligned}
$$

Now, upon expanding the left-hand side of (15), we have

$$
\begin{aligned}
& \frac{z\left(D_{q} \mathcal{I}_{q}^{\lambda} f\right)(z)}{f(z)}=1+q \psi_{1} a_{2} z+\left\{\left(q+q^{2}\right) \psi_{2} a_{3}-q \psi_{1}^{2} a_{2}^{2}\right\} z^{2} \\
& \quad+\left\{\left(q+q^{2}+q^{3}\right) \psi_{3} a_{4}-\left(2 q+q^{2}\right) \psi_{1} \psi_{2} a_{2} a_{3}+q \psi_{1}^{3} a_{2}^{3}\right\} z^{3}+\cdots
\end{aligned}
$$

Finally, by comparing the corresponding coefficients in (17) and (18) along with Lemma 3, we obtain the result asserted by Theorem 1.

Theorem 2. If the function $f(z)$ given by (1) belongs to the class $\mathcal{S T}(k, \lambda, q)$, then

$$
\begin{aligned}
\mathcal{T}_{3}(2) \leqq & {\left[\left(\frac{1+q}{2 q \psi_{1}}\right) p_{1}^{2}+\left(\frac{1+q}{4\left(q+q^{2}+q^{3}\right) \psi_{3}}\right)\left[\Omega_{1}+\Omega_{2}\right]\right] } \\
& \cdot\left[4\left(\frac{(1+q)^{2}}{16 q^{2} \psi_{1}^{2}}\right) p_{1}^{2}+16\left|\Omega_{3}\right|+\frac{p_{1}^{2}}{4 q^{2} \psi_{2}^{2}}+2 \Omega_{5} p_{1}^{2}\left|2-\frac{\Omega_{4}}{\Omega_{5} p_{1}^{2}}\right|\right],
\end{aligned}
$$


where

$$
\begin{aligned}
\Omega_{1}= & 2 p_{1}+4\left|p_{2}-p_{1}+\frac{\left(2+q^{2}\right)}{4 q} p_{1}^{2}\right|, \\
\Omega_{2}= & \mid 2 p_{3}+2 p_{1}-4 p_{2}-\left(2\left(1+q^{2}\right)-q\right) p_{1}^{2} \\
& +\left(\frac{4 q^{2}-3 q+2}{q}\right) p_{1} p_{2}+\left(\frac{q^{2}+q+1}{2 q^{2}} p_{1}^{3}\right) \mid, \\
\Omega_{3}= & \frac{1}{2 q^{2} \psi_{2}^{2}}\left(\frac{p_{2}}{4}-\frac{p_{1}}{4}+\frac{\left(q^{2}+1\right) p_{1}^{2}}{8 q}\right)^{2}-\Omega_{5} \cdot\left[\frac{p_{3}}{4}+\frac{p_{1}}{4}-\frac{p_{2}}{2}\right. \\
& \left.\quad-\frac{\left[2\left(1+q^{2}\right)-q\right] p_{1}^{2}}{8 q}+\frac{4 q^{2}-3 q+2}{8 q} p_{1} p_{2}+\left(\frac{q^{2}+2 q-1}{16 q^{2}}\right) p_{1}^{3}\right], \\
\Omega_{4}= & \frac{p_{1}}{2 q^{2} \psi_{2}^{2}}\left(\frac{p_{2}}{4}-\frac{p_{1}}{4}+\frac{\left(q^{2}+1\right) p_{1}^{2}}{8 q}\right)-\Omega_{5} p_{1}\left(p_{2}-p_{1}+\frac{\left(2+q^{2}\right) p_{1}^{2}}{4 q}\right), \\
\Omega_{5}= & \frac{(1+q)^{2}}{16 q^{2}\left(1+q+q^{2}\right) \psi_{1} \psi_{3}}
\end{aligned}
$$

and $p_{j}(j=1,2)$ are positive and are the coefficients of the functions $p_{k}(z)$ defined by (6).

Proof. Upon comparing the corresponding coefficients in (17) and (18), we find that

$$
\begin{aligned}
a_{2}= & \frac{(1+q) p_{1} c_{1}}{4 q \psi_{1}} \\
a_{3}= & \frac{1}{2 q \psi_{2}}\left[\frac{p_{1} c_{2}}{2}+\left(\frac{p_{2}}{4}-\frac{p_{1}}{4}+\frac{\left(q^{2}+1\right) p_{1}^{2}}{8 q}\right) c_{1}^{2}\right] \\
a_{4}= & \frac{(1+q)}{4\left(q+q^{2}+q^{3}\right) \psi_{3}}\left[p_{1} c_{3}+\left(p_{2}-p_{1}+\frac{\left(2+q^{2}\right) p_{1}^{2}}{4 q}\right) c_{1} c_{2}\right. \\
& +\left(\frac{p_{3}}{4}+\frac{p_{1}}{4}-\frac{p_{2}}{2}-\frac{\left(2\left(1+q^{2}\right)-q\right) p_{1}^{2}}{8 q}+\frac{\left(4 q^{2}-3 q+2\right)}{8 q} p_{1} p_{2}\right. \\
& \left.\left.+\frac{\left(q^{2}+2 q-1\right)}{16 q^{2}} p_{1}^{3}\right) c_{1}^{3}\right] .
\end{aligned}
$$

By a simple computation, $\mathcal{T}_{3}(2)$ can be written as follows:

$$
\mathcal{T}_{3}(2)=\left(a_{2}-a_{4}\right)\left(a_{2}^{2}-2 a_{3}^{2}+a_{2} a_{4}\right) .
$$

Now, if $f \in \mathcal{S T}(k, \lambda, q)$, then it is clearly seen that

$$
\begin{aligned}
\left|a_{2}-a_{4}\right| & \leqq\left|a_{2}\right|+\left|a_{4}\right| \\
& \leqq\left(\frac{1+q}{2 q \psi_{1}}\right) p_{1}^{2}+\left(\frac{1+q}{4\left(q+q^{2}+q^{3}\right) \psi_{3}}\right)\left(\Omega_{1}+\Omega_{2}\right) .
\end{aligned}
$$


We need to maximize $\left|a_{2}^{2}-2 a_{3}^{2}+a_{2} a_{4}\right|$ for a function $f \in \mathcal{S T}(k, \lambda, q)$. So, by writing $a_{2}, a_{3}$, and $a_{4}$ in terms of $c_{1}, c_{2}$, and $c_{3}$, with the help of (19)-(21), we get

$$
\begin{aligned}
\mid a_{2}^{2}- & 2 a_{3}^{2}+a_{2} a_{4} \mid \\
= & \left|\left(\frac{(1+q)^{2}}{16 q^{2} \psi_{1}^{2}}\right) p_{1}^{2} c_{1}^{2}-\Omega_{3} c_{1}^{4}-\Omega_{4} c_{1}^{2} c_{2}-\frac{p_{1}^{2}}{8 q^{2} \psi_{2}^{2}} c_{2}^{2}+\Omega_{5} p_{1}^{2} c_{1} c_{3}\right| .
\end{aligned}
$$

Finally, by applying the trigonometric inequalities, Lemmas 2 and 3 along with (22), we obtain the result asserted by Theorem 2 .

As an application of Theorem 2, we first set $\psi_{n-1}=1$ and $k=0$ and then let $q \rightarrow 1-$. We thus arrive at the following known result.

Corollary 1 (see [25]). If the function $f(z)$ given by (1) belongs to the class $\mathcal{S}^{*}$, then

$$
\mathcal{T}_{3}(2) \leqq 84 \text {. }
$$

Theorem 3. If the function $f(z)$ given by (1) belongs to the class $\mathcal{S T}(k, \lambda, q)$, then

$$
\left|a_{2} a_{4}-a_{3}^{2}\right| \leqq \frac{1}{4 q^{2} \psi_{2}^{2}} p_{1}^{2},
$$

where $k \in[0,1]$ and $p_{j}(j=1,2,3)$ are positive and are the coefficients of the functions $p_{k}(z)$ defined by $(6)$.

Proof. Making use of (19)-(21), we find that

$$
\begin{aligned}
a_{2} a_{4}-a_{3}^{2} & =\frac{A(q)}{16 q^{2} \psi_{1} \psi_{3}} p_{1}^{2} c_{1} c_{3}+\left(\frac{A(q) \psi_{2}^{2}-\psi_{1} \psi_{3}}{16 q^{2} \psi_{1} \psi_{2}^{2} \psi_{3}} p_{1} p_{2}-\frac{A(q) \psi_{2}^{2}-\psi_{1} \psi_{3}}{16 q^{2} \psi_{1} \psi_{2}^{2} \psi_{3}} p_{1}^{2}\right. \\
& \left.+\frac{A(q)\left(2+q^{2}\right) \psi_{2}^{2}-2\left(1+q^{2}\right) \psi_{1} \psi_{3}}{64 q^{2} \psi_{1} \psi_{3}} p_{1}^{3}\right) c_{1}^{2} c_{2}+\frac{1}{16 q^{2} \psi_{2}^{2}} p_{1}^{2} c_{2}^{2} \\
& +\left[\frac{A(q)}{64 q^{2} \psi_{1} \psi_{3}} p_{1} p_{3}+\left(\frac{A(q) \psi_{2}^{2}-\psi_{1} \psi_{3}}{64 q^{2} \psi_{1} \psi_{2}^{2} \psi_{3}}\right) p_{1}^{2}+\left(\frac{\psi_{1} \psi_{3}-A(q) \psi_{2}^{2}}{32 q^{2} \psi_{1} \psi_{2}^{2} \psi_{3}}\right) p_{1} p_{2}\right. \\
& +\left(\frac{2\left(1+q^{2}\right) \psi_{1} \psi_{3}-\left(2\left(1+q^{2}\right)-q\right) A(q) \psi_{2}^{2}}{128 q^{3} \psi_{1} \psi_{2}^{2} \psi_{3}}\right) p_{1}^{3} \\
& +\left(\frac{A(q)\left(4 q^{2}-3 q+2\right) \psi_{2}^{2}-2\left(1+q^{2}\right) \psi_{1} \psi_{3}}{128 q^{3} \psi_{1} \psi_{2}^{2} \psi_{3}}\right) p_{1}^{2} p_{2} \\
& \left.+\left(\frac{A(q)\left(q^{2}+2 q-1\right) \psi_{2}^{2}-\left(1+q^{2}\right)^{2} \psi_{1} \psi_{3}}{256 q^{4} \psi_{1} \psi_{2}^{2} \psi_{3}}\right) p_{1}^{4}-\frac{1}{64 q^{2} \psi_{2}^{2}} p_{2}^{2}\right] c_{1}^{4},
\end{aligned}
$$

where

$$
A(q)=\frac{(1+q)^{2}}{1+q+q^{2}} .
$$


We substitute the values of $c_{2}$ and $c_{3}$ from the above Lemma and, for simplicity, take $Y=4-c_{1}^{2}$ and $Z=\left(1-|x|^{2}\right) z$. Without loss of generality, we assume that $c=c_{1}(0 \leqq c \leqq 2)$, so that

$$
\begin{aligned}
a_{2} a_{4}-a_{3}^{2}= & {\left[\frac{q(1-q) A(q) \psi_{2}^{2}}{128 q^{2} \psi_{1} \psi_{3}} p_{1}^{3}+\frac{A(q)}{64 q^{2} \psi_{1} \psi_{3}} p_{1} p_{3}\right.} \\
+ & \left(\frac{A(q)\left(4 q^{2}-3 q+2\right) \psi_{2}^{2}-2\left(1+q^{2}\right) \psi_{1} \psi_{3}}{128 q^{3} \psi_{1} \psi_{2}^{2} \psi_{3}}\right) p_{1}^{2} p_{2} \\
+ & \left.\left(\frac{A(q)\left(q^{2}+2 q-1\right) \psi_{2}^{2}-\left(1+q^{2}\right)^{2} \psi_{1} \psi_{3}}{256 q^{4} \psi_{1} \psi_{2}^{2} \psi_{3}}\right) p_{1}^{4}-\frac{1}{64 q^{2} \psi_{2}^{2}} p_{2}^{2}\right] c^{4} \\
+ & {\left[\frac{A(q) \psi_{2}^{2}-\psi_{1} \psi_{3}}{32 q^{2} \psi_{1} \psi_{2}^{2} \psi_{3}} p_{1} p_{2}+\frac{A(q)\left(2+q^{2}\right) \psi_{2}^{2}-2\left(1+q^{2}\right) \psi_{1} \psi_{3}}{128 q^{2} \psi_{1} \psi_{3}} p_{1}^{3}\right] c^{2} x Y } \\
& \cdot\left[-\frac{A(q)}{64 q^{2} \psi_{1} \psi_{3}} p_{1}^{2} c^{2} Y x^{2}-\frac{1}{64 q^{2} \psi_{2}^{2}} p_{1}^{2} x^{2} Y^{2}+\frac{A(q)}{32 q^{2} \psi_{1} \psi_{3}} p_{1}^{2} c Y Z\right] .
\end{aligned}
$$

Upon setting $Z=\left(1-|x|^{2}\right) z$ and taking the moduli in (25) and using trigonometric inequality, we find that

$$
\begin{aligned}
\left|a_{2} a_{4}-a_{3}^{2}\right| & \leqq\left|\lambda_{1}\right| c^{4}+\left|\lambda_{2}\right||x| Y c^{2}+\frac{A(q)}{64 q^{2} \psi_{1} \psi_{3}} p_{1}^{2} Y|x|^{2} c^{2} \\
& +\frac{1}{64 q^{2} \psi_{2}^{2}} p_{1}^{2}|x|^{2} Y^{2}+\frac{A(q)}{32 q^{2} \psi_{1} \psi_{3}} p_{1}^{2} c^{2} Y\left(1-|x|^{2}\right) \\
= & \Lambda(c,|x|),
\end{aligned}
$$

where

$$
\begin{aligned}
\lambda_{1} & =\frac{q(1-q) A(q) \psi_{2}^{2}}{128 q^{2} \psi_{1} \psi_{3}} p_{1}^{3}+\frac{A(q)}{64 q^{2} \psi_{1} \psi_{3}} p_{1} p_{3} \\
& +\left(\frac{A(q)\left(4 q^{2}-3 q+2\right) \psi_{2}^{2}-2\left(1+q^{2}\right) \psi_{1} \psi_{3}}{128 q^{3} \psi_{1} \psi_{2}^{2} \psi_{3}}\right) p_{1}^{2} p_{2} \\
& +\left(\frac{A(q)\left(q^{2}+2 q-1\right) \psi_{2}^{2}-\left(1+q^{2}\right)^{2} \psi_{1} \psi_{3}}{256 q^{4} \psi_{1} \psi_{2}^{2} \psi_{3}}\right) p_{1}^{4}-\frac{1}{64 q^{2} \psi_{2}^{2}} p_{2}^{2} \\
\lambda_{2} & =\frac{A(q) \psi_{2}^{2}-\psi_{1} \psi_{3}}{32 q^{2} \psi_{1} \psi_{2}^{2} \psi_{3}} ; p_{1} p_{2}+\frac{A(q)\left(2+q^{2}\right) \psi_{2}^{2}-2\left(1+q^{2}\right) \psi_{1} \psi_{3}}{128 q^{2} \psi_{1} \psi_{3}} p_{1}^{3} .
\end{aligned}
$$

Now, trivially, we have

$$
\Lambda^{\prime}(|x|)>0
$$

on $[0,1]$, and so

$$
\Lambda(|x|) \leqq \Lambda(1) .
$$

Hence, by puting $Y=4-c_{1}^{2}$ and after some simplification, we have

$$
\begin{aligned}
\left|a_{2} a_{4}-a_{3}^{2}\right|= & \left(\left|\lambda_{1}\right|-\left|\lambda_{2}\right|+\frac{\psi_{1} \psi_{3}-A(q) \psi_{2}^{2}}{64 q^{2} \psi_{1} \psi_{3}} p_{1}^{2}\right) c^{4} \\
& +\left(4\left|\lambda_{2}\right|+\left(\frac{A(q) \psi_{2}^{2}-\psi_{1} \psi_{3}}{16 q^{2} \psi_{1} \psi_{3}} p_{1}^{2}\right)\right) c^{2}+\frac{1}{4 q^{2} \psi_{2}^{2}} p_{1}^{2} \\
= & G(c) .
\end{aligned}
$$


For optimum value of $G(c)$, we consider $G^{\prime}(c)=0$, which implies that $c=0$. So $G(c)$ has a maximum value at $c=0$. We therefore conclude that the maximum value of $G(c)$ is given by

$$
\frac{1}{4 q^{2} \psi_{2}^{2}} p_{1}^{2}
$$

which occurs at $c=0$ or

$$
c^{2}=-\frac{128\left|\lambda_{2}\right| q^{2} \psi_{1} \psi_{3}+4 A(q) \psi_{2}^{2}-2 \psi_{1} \psi_{3} p_{1}^{2}}{\left(64 q^{2}\left(\left|\lambda_{1}\right|-\left|\lambda_{2}\right|\right) \psi_{1} \psi_{3}+\psi_{1} \psi_{3}-A(q) \psi_{2}^{2} p_{1}^{2}\right)} .
$$

This completes the proof of Theorem 3.

If we put $\psi_{n-1}=1$ and let $q \rightarrow 1-$ in Theorem 3, we have the following known result.

Corollary 2 (see [26]). If the function $f(z)$ given by (1) belongs to the class $k-\mathcal{S} \mathcal{T}$, where $k \in[0,1]$, then

$$
\left|a_{2} a_{4}-a_{3}^{2}\right| \leqq \frac{p_{1}^{2}}{4}
$$

If we put

$$
p_{1}=2 \text { and } \psi_{n-1}=1,
$$

by letting $q \rightarrow 1-$ in Theorem 3 , we have the following known result.

Corollary 3 (see [18]). If $f \in \mathcal{S}^{*}$, then

$$
\left|a_{2} a_{4}-a_{3}^{2}\right| \leqq 1
$$

By letting $k=1, \psi_{n-1}=1, q \rightarrow 1-$ and

$$
p_{1}=\frac{8}{\pi^{2}}, \quad p_{2}=\frac{16}{3 \pi^{2}} \text { and } p_{3}=\frac{184}{45 \pi^{2}}
$$

in Theorem 3, we have the following known result.

Corollary 4 (see [27]). If the function $f(z)$ given by (1) belong to the class $\mathcal{S P}$, then

$$
\left|a_{2} a_{4}-a_{3}^{2}\right| \leqq \frac{16}{\pi^{4}}
$$

\section{Concluding Remarks and Observations}

Motivated significantly by a number of recent works, we have made use of a certain general conic domain and the quantum (or $q$-) calculus in order to define and investigate a new subclass of normalized analytic functions in the open unit disk $\mathbb{U}$, which we have referred to as $q$-starlike functions. For this $q$-starlike function class, we have successfully derived several properties and characteristics. In particular, we have found the Hankel determinant and the Toeplitz matrices for this newly-defined class of $q$-starlike functions. We also highlight some known consequences of our main results which are stated and proved as theorems and corollaries.

Author Contributions: conceptualization, Q.Z.A. and N.K. (Nazar Khan); methodology, N.K. (Nasir Khan); software, B.K.; validation, H.M.S.; formal analysis, H.M.S.; writing-original draft preparation, H.M.S.; writing-review and editing, H.M.S.; supervision, H.M.S.

Funding: This research received no external funding.

Conflicts of Interest: The authors declare no conflict of interest. 


\section{References}

1. Miller, S.S.; Mocanu, P.T. Differential subordination and univalent functions. Mich. Math. J. 1981, $28,157-171$. [CrossRef]

2. Miller, S.S.; Mocanu, P.T. Differential Subordination: Theory and Applications; Series on Monographs and Textbooks in Pure and Applied Mathematics, No. 225; Marcel Dekker Incorporated: New York, NY, USA; Basel, Switzerland, 2000.

3. Kanas, S.; Wiśniowska, A. Conic regions and k-uniform Convexity. J. Comput. Appl. Math. 1999, 105, 327-336. [CrossRef]

4. Kanas, S.; Wiśniowska, A. Conic domains and starlike Functions. Rev. Roum. Math. Pures Appl. 2000, 45, 647-657.

5. Kanas, S.; Srivastava, H.M. Linear operators associated with $k$-uniformly convex functions. Integral Transform. Spec. Funct. 2000, 9, 121-132. [CrossRef]

6. Mahmood, S.; Jabeen, M.; Malik, S.N.; Srivastava, H.M.; Manzoor, R.; Riaz, S.M.J. Some coefficient inequalities of $q$-starlike functions associated with conic domain defined by $q$-derivative. J. Funct. Spaces 2018, 2018, 8492072. [CrossRef]

7. Srivastava, H.M.; Karlsson, P.W. Multiple Gaussian Hypergeometric Series; Ellis Horwood Limited: Chichester, UK, 1985.

8. Jackson, F.H. On q-definite integrals. Quart. J. Pure Appl. Math. 1910, 41, 193-203.

9. Jackson, F.H. q-difference equations. Am. J. Math. 1910, 32, 305-314. [CrossRef]

10. Kanas, S.; Răducanu, D. Some class of analytic functions related to conic domains. Math. Slov. 2014, 64, 1183-1196. [CrossRef]

11. Noor, K.I. On new classes of integral operators. J. Nat. Geom. 1999, 16, 71-80.

12. Ismail, M.E.H.; Merkes, E.; Styer, D. A generalization of starlike functions. Complex Var. Theory Appl. 1990, 14, 77-84. [CrossRef]

13. Srivastava, H.M. Univalent functions, fractional calculus, and associated generalized hypergeometric functions. In Univalent Functions, Fractional Calculus and Their Applications; Srivastava, H.M., Owa, S., Eds.; Ellis Horwood Limited: Chichester, UK, 1989; pp. 329-354.

14. Srivastava, H.M.; Bansal, D. Close-to-convexity of a certain family of $q$-Mittag-Leffer functions. J. Nonlinear Var. Anal. 2017, 1, 61-69.

15. Uçar, H.E.Ö. Coefficient inequality for $q$-starlike Functions. Appl. Math. Comput. 2016, 76, 122-126.

16. Sahoo, S.K.; Sharma, N.L. On a generalization of close-to-convex functions. Ann. Polon. Math. 2015, 113, 93-108. [CrossRef]

17. Noonan, J.W.; Thomas, D.K. On the second Hankel derminant of areally mean $p$-valent functions. Trans. Am. Math. Soc. 1976, 223, 337-346.

18. Janteng, A.; Abdul-Halim, S.; Darus, M. Hankel determinant for starlike and convex functions. Int. J. Math. Anal. 2007, 1, 619-625.

19. Mishra, A.K.; Gochhayat, P. Second Hankel determinant for a class of analytic functions defined by fractional derivative. Internat. J. Math. Math. Sci. 2008, 2008, 153280. [CrossRef]

20. Singh, G.; Singh, G. On the second Hankel determinant for a new subclass of analytic functions. J. Math. Sci. Appl. 2014, 2, 1-3.

21. Babalola, K.O. On $\mathrm{H}_{3}(1)$ Hankel determinant for some classes of univalent functions. Inequal. Theory Appl. 2007, 6, 1-7.

22. Duren, P.L. Univalent Functions (Grundlehren der Mathematischen Wissenschaften 259); Springer: New York, NY, USA; Berlin/Heidelberg, Germany; Tokyo, Japan, 1983.

23. Thomas, D.K.; Abdul-Halim, S. Toeplitz matrices whose elements are the coefficients of starlike and close-to-convex functions. Bull. Malays. Math. Sci. Soc. 2017, 40, 1781-1790. [CrossRef]

24. Efraimidis, I. A generalization of Livingston's coefficient inequalities for functions with positive real part. J. Math. Anal. Appl. 2016, 435, 369-379. [CrossRef]

25. Ali, M.F.; Thomas, D.K.; Vasudevarao, A. Toeplitz determinants whose element are the coefficients of univalent functions. Bull. Aust. Math. Soc. 2018, 97, 253-264. [CrossRef] 
26. Ramachandran, C.; Annamalai, S. On Hankel and Toeplitz determinants for some special class of analytic functions involving conical domains defined by subordination. Internat. J. Engrg. Res. Technol. 2016, 5, 553-561.

27. Lee, S.K.; Ravichandran, V.; Supramaniam, S. Bounds for the second Hankel determinant of certain univalent functions. J. Inequal. Appl. 2013, 2013, 281-297. [CrossRef]

(C) 2019 by the authors. Licensee MDPI, Basel, Switzerland. This article is an open access article distributed under the terms and conditions of the Creative Commons Attribution (CC BY) license (http:/ / creativecommons.org/licenses/by/4.0/). 